\title{
Neutrophil infiltration favors colitis-associated tumorigenesis by activating the interleukin-1 $(\mathrm{IL}-1) / \mathrm{IL}-6$ axis
}

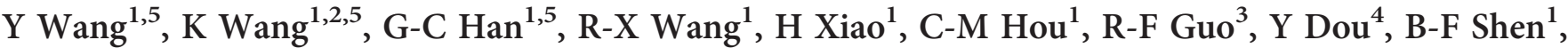 \\ $\mathrm{Y} \mathrm{Li}^{1}$ and G-J Chen ${ }^{1}$
}

Neutrophil infiltration is a key event in chronic intestinal inflammation and associated colorectal cancer, but how these cells support cancer development is poorly understood. In this study, using a mouse model of colitis-associated cancer (CAC), we have demonstrated that infiltrated neutrophils produce large amounts of interleukin-1 (IL)-1 $\beta$ that is critical for the development of CAC. Depletion of neutrophil or blockade of IL-1 $\beta$ activity significantly reduced mucosal damage and tumor formation. This protumorigenic function of IL-1 $\beta$ was mainly attributed to increased IL- 6 secretion by intestineresident mononuclear phagocytes (MPs). Furthermore, commensal flora-derived lipopolysaccharide (LPS) was identified to trigger IL-1 $\beta$ expression in neutrophils. Importantly, accumulation of IL-1 $\beta$-expressing neutrophils was seen in lesions of patients suffering from ulceratic $\mathrm{CAC}$ and these infiltrated neutrophils induced IL-6 production by intestinal MPs in an IL-1 $\beta$-dependent manner. Overall, these findings reveal that in CAC milieu, infiltrating neutrophils secrete IL-1 $\beta$ that promotes tumorigenesis by inducing IL- 6 production by intestinal MPs.

\section{INTRODUCTION}

It is well established that chronic inflammation is a major driving force for tumor promotion and progression in many organs. ${ }^{1}$ For instance, in patients with ulcerative colitis, one of the major forms of human chronic intestinal bowel disease, the risk of developing colorectal cancer is higher than in the general population. ${ }^{2,3}$ It has been proposed that dysregulated intestinal homeostasis, characterized by mucosal damage and microbiota translocation, contributes to colon carcinogenesis by recruiting proinflammatory immune cells (e.g., tumor-associated macrophage) into lesions, where they produce tumor-promoting cytokines that make a major contribution to colon cancer growth. ${ }^{4}$ Recent studies have demonstrated that in the colitisassociated cancer (CAC) microenvironment, intestinal myeloid cells (predominantly dendritic cells (DCs) and macrophages) produce large amounts of interleukin-6 (IL-6) and promote tumor growth via the nuclear factor- $\kappa \mathrm{B}$-dependent signaling pathway and that the protumor effect of IL-6 is related to its ability to enhance proliferation and decrease apoptosis of preneoplastic intestinal epithelial cells (IECs). ${ }^{5,6}$ How this cytokine production is regulated during CAC tumorigenesis remains unclear.

The role in cancer biology of the neutrophil, the most abundant cell involved in innate immunity, has been recently highlighted. ${ }^{78}$ In tumor-bearing mice, the neutrophil is thought to be in a protumorigenic state. ${ }^{9}$ The mechanisms responsible for this protumor activity have not yet been fully elucidated, but neutrophils have an impact on angiogenesis, immune surveillance, and the secretion of chemokines, cytokines, and reactive oxygen species. ${ }^{8}$ During intestinal inflammation, the neutrophil has been reported to induce cell arrest and replication errors in colonic epithelial cells or to produce carcinogenic $\mathrm{N}$-nitroso compounds, thus facilitating colon carcinogenesis. ${ }^{10}$ A recent study demonstrated that neutrophils play a pathogenic role in the development of CAC, ${ }^{11}$ but the mechanism is still largely unknown. Here we show that infiltrating neutrophils in

\footnotetext{
${ }^{1}$ Department of Immunology, Institute of Basic Medical Sciences, Beijing, People's Republic of China. ${ }^{2}$ Department of Immunology, School of Basic Medical Sciences, Central South University, Changsha, People's Republic of China. ${ }^{3}$ Department of Pathology, University of Michigan, Ann Arbor, Michigan, USA and ${ }^{4}$ Department of Gastroenterology, General Hospital of the PLA, Beijing, People's Republic of China. Correspondence: G-J Chen (guogangch@yahoo.com)

${ }^{5}$ These authors contributed equally to this work.
} 
CAC lesions abundantly produce proinflammatory cytokine IL-1 $\beta$. Furthermore, we identify a protumor role of neutrophil/ IL-1 $\beta$-MPs/IL-6 axis in CAC development.

\section{RESULTS}

\section{Neutrophils infiltrate CAC lesions and contribute to CAC development}

First, the involvement of neutrophil in CAC carcinogenesis was examined in both mouse experimental model and patients. The mouse model was induced in male Balb/c or TLR4 - / mice aged 6-8 weeks by intraperitoneal injection of azoxymethane, followed by 4 cycles ( 84 days total) of dextran sulfate sodium (DSS) treatment, and examination on day 100 .
Numerous neutrophil infiltrates were observed in lesions in the mouse model (Figure 1a and Supplementary Figure S1 online) and in specimens from CAC patients (Supplementary Figure S2), suggesting a tight relationship between neutrophil infiltration and CAC.

To determine the role of neutrophils in CAC tumorigenesis, we depleted this population in our mouse model using antibodies recognizing Ly-6G that is highly and specifically expressed in neutrophils, ${ }^{12}$ with the efficacy of depletion being confirmed by fluorescence-activated cell sorting analysis (Supplementary Figure S3). Depletion of neutrophils led to attenuation of mucosal damage and a reduced tumor load in mice with induced CAC (Figure 1b,c) and to increased
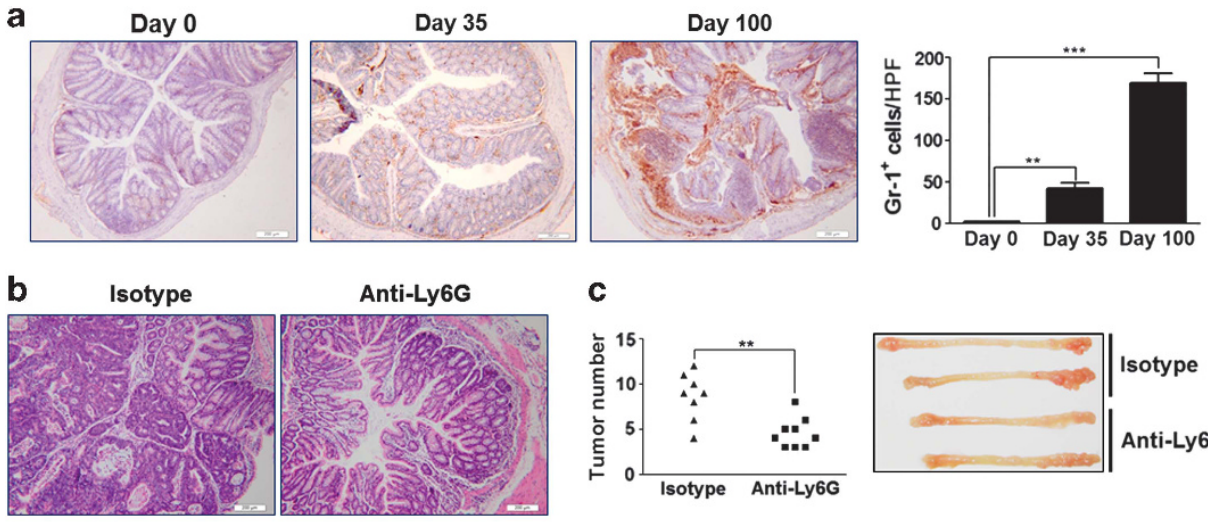

C
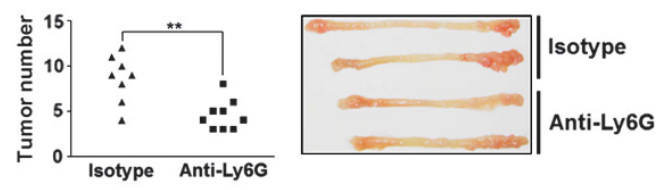

d
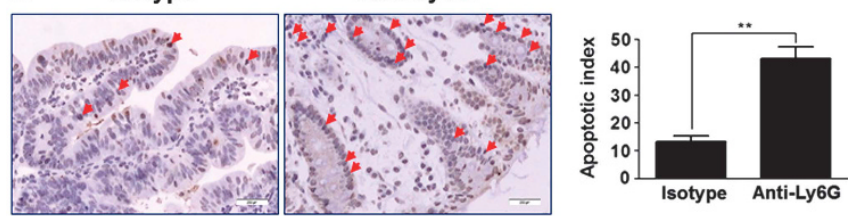

e

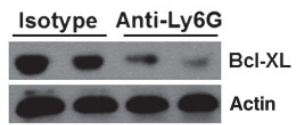

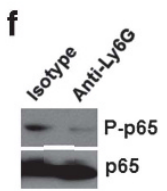
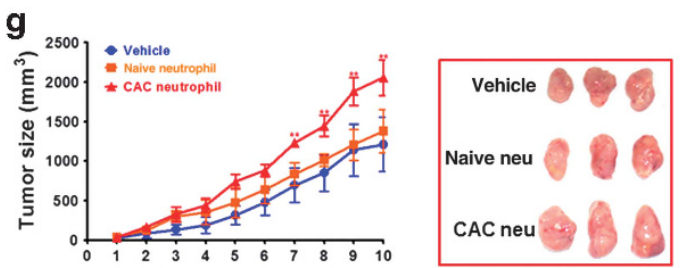

Day after tumor inoculation

Figure 1 Neutrophils infiltrate into colitis-associated cancer (CAC) lesions and contribute to tumor growth. (a) The colon tissues of mice with or without CAC were removed at days 35 and 100 respectively and fixed with formalin and then processed conventionally. Infiltrating neutrophils were identified by staining with anti-Gr-1 antibody (brown). Six pairs of mice were analyzed. Scale bar $=200 \mu \mathrm{m}$. The cell number per field was counted in five randomly selected visual fields at original magnification $\times 400$. Representative results of six mice are shown on the right panel. HPF, high-power field. (b) Mice about to undergo CAC induction were injected intraperitoneally with anti-Ly6G antibody or isotype control on days -3 and -2 before initiation of dextran sulfate sodium (DSS) drinking (day 0), and then twice a week (days 2 and 5 each week) throughout the entire experimental period. Colon tissues were isolated on day 100 and stained with hematoxylin and eosin (H\&E). Eight pairs of mice were analyzed. Scale bar $=200 \mu \mathrm{m}$. (c) Mice were treated as in b, and then the number of tumors in the colon was counted (left). Representative images of colon tissues are shown on the right. (d) Colon tissues of mice treated as in b were isolated and TUNEL (TdT-mediated dNTP nick end labeling) stained (brown) for apoptosis of intestinal epithelial cells (IECs). Five pairs of mice were examined. An example is shown on the left and the summarized results on the right. The arrows denote the apoptotic cells. Scale bar $=200 \mu \mathrm{m}$. (e) IECs were isolated from the colons of mice treated as in $\mathbf{b}$ and proteins were extracted and Bcl-XL (B-cell lymphoma-extra large) levels were measured by western blotting. The data shown are representative of those obtained in two independent experiments. (f) IECs were isolated from the colons of mice treated as in $\mathbf{b}$ and proteins were extracted, and then phosphorylation of the nuclear factor (NF)- $\mathrm{kB}$ p65 subunit was detected by western blotting. The data shown are representative of those obtained in three independent experiments. (g) Neutrophils in the lesions of CAC-bearing mice or in the spleen of naive littermates were isolated by fluorescence-activated cell sorting (FACS) and then implanted into transplanted CT-26 tumors in nude mice. Control nude mice implanted with tumors were injected with phosphate-buffered saline (PBS). Tumor size was monitored every day. Each group consisted of 6-8 mice. Left, summarized results; right, representative images of transplanted tumor tissues. ${ }^{\star \star} P<0.01 ;{ }^{\star \star \star} P<0.001$. 
apoptosis of IECs (Figure 1d). As the antiapoptotic protein BclXL (B-cell lymphoma-extra large) has been suggested to be important for IEC survival, ${ }^{13}$ we examine its expression in IECs and found that, in neutrophil-depleted mice, IECs expressed less Bcl-XL (Figure 1e). Furthermore, activation of nuclear factor- $\kappa B$ in IECs was inhibited in the neutrophil-depleted mice (Figure 1f) that might account for the increase in IEC death. To further examine the tight association between infiltrating neutrophils and tumor growth, we prepared neutrophils from naive and CAC-bearing mice and determined their protumor function in a xenograft tumor model, and found that infusion of neutrophils from lesions of CAC-bearing mice, but not their naive counterparts, accelerated tumor growth (Figure 1g). It is noteworthy that transferred neutrophils were alive at the intervals of observation (Supplementary Figure S4), indicating that the neutrophil-mediated protumor effects were not secondary to the autophagy of these cells. Taken together, these findings show that, by controlling the apoptosis and proliferation of transformed IECs, infiltrating neutrophils are required for colon tumorigenesis in the CAC model.

\section{Neutrophils that infiltrate the intestine in CAC-bearing mice are the main source of IL-1 $\beta$}

Although it is well established that IL-1 signaling is required for CAC development, ${ }^{14,15}$ the cellular source of IL-1 $\beta$ in the CAC microenvironment is not known. To address this issue, we measured IL-1 $\beta$ contents in colonic tissues and observed an increase following the establishment of CAC (Figure 2a). To identify the cell type producing IL-1 $\beta$, four subpopulations of lamina propria (LP) immune cells were prepared on the basis of Gr-1 and CD11b staining and their IL-1 $\beta$ mRNA levels measured. As shown in Figure 2b, the results showed that the $\mathrm{Gr}-1^{\text {hi }} \mathrm{CD} 11 \mathrm{~b}^{+}$cells (neutrophils) had the highest IL-1 $\beta$ mRNA levels before and after CAC induction and expressed much more IL-1 $\beta$ mRNA following establishment of CAC. Intriguingly, IL-1 $\beta$ mRNA expression was also detected in $\mathrm{Gr}-1^{\mathrm{lo} /}-\mathrm{CD} 11 \mathrm{~b}^{+}$cells (DCs and macrophages), although at much lower levels (Figure $2 \mathbf{b}$ ). In accord with this, infiltrating neutrophils from CAC-bearing mice were found to contain more IL- $1 \beta$ protein than their naive littermates (Figure 2c). Interestingly, neutrophils from the spleen of CAC-bearing mice expressed much lower amounts of IL-1 $\beta$ mRNA that were comparable to those in controls (Figure 2d). These findings indicate that the intestinal inflammatory milieu elicits high IL-1 $\beta$ expression in infiltrating neutrophils and that this is critical for CAC development.

\section{In vivo blockade of IL-1 $\beta$ activity in mice reduces CAC carcinogenesis}

To determine whether IL-1 $\beta$ was involved in CAC development, we used the IL-1 receptor antagonist (IL-1Ra) to block
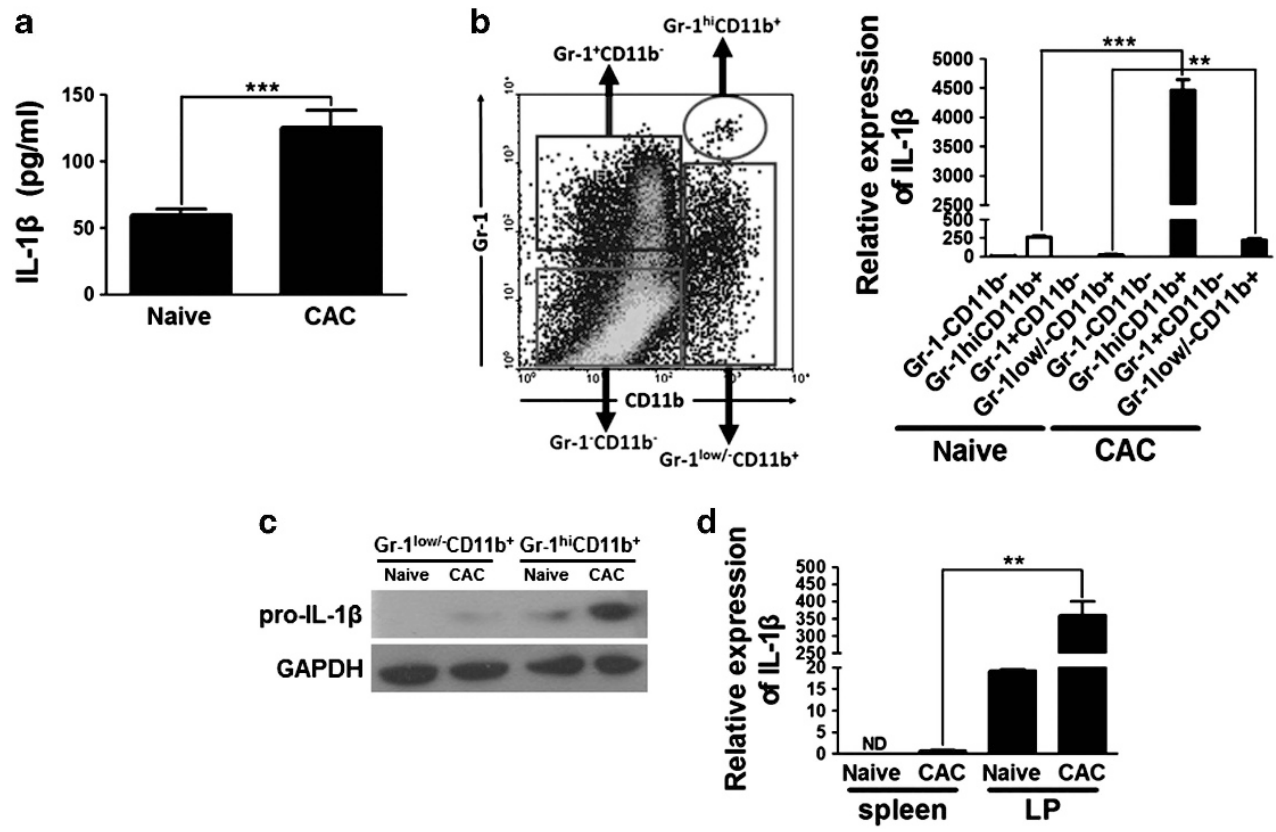

Figure 2 Neutrophils infiltrating into colitis-associated cancer (CAC) lesions highly express interleukin-1 $\beta$ (IL-1 $\beta$ ). (a) Colon tissues from CAC-bearing mice or naive littermates were dissected and cultured in serum-free medium for $24 \mathrm{~h}$, and then the supernatants were collected and IL-1 $\beta$ levels were measured by enzyme-linked immunosorbent assay (ELISA). Ten pairs of mice were analyzed. (b) Immune cells in the lamina propria (LP) of CAC-bearing or naive mice were sorted by staining for Gr-1 and CD11b and divided into four subpopulations (left panel): Gr- $1^{-} \mathrm{CD} 11 \mathrm{~b}^{-}, \mathrm{Gr}-1^{\text {hi }} \mathrm{CD} 11 \mathrm{~b}{ }^{+}, \mathrm{Gr}-$ $1^{+} \mathrm{CD}_{11} \mathrm{~b}^{-}$, and Gr-1 $1^{\mathrm{low} /-} \mathrm{CD} 11 \mathrm{~b}^{+}$, and then IL-1 $\beta$ mRNA levels in these cells were determined by quantitative reverse-transcriptase-polymerase chian reaction (RT-PCR). Six pairs of mice were analyzed. (c) LP-residing Gr- $1^{\text {hi }} C D 11 b^{+}$and Gr- $1^{\text {low } /-}$ CD $11 b^{+}$subsets were sorted as above and lysed, and then pro-IL-1 $\beta$ levels were determined by western blotting. The data shown are representative of those obtained in five independent experiments. (d) Spleen and colon tissues of CAC-bearing or naive mice were isolated and neutrophils $\left(\mathrm{Gr}-1^{\text {hi }} \mathrm{CD} 11 \mathrm{~b}^{+}\right)$in these tissues sorted by fluorescence-activated cell sorting (FACS), and then IL-1 $\beta$ mRNA levels were determined by quantitative RT-PCR. Five pairs of mice were analyzed. GAPDH, glyceraldehyde-3-phosphate dehydrogenase; ND, not detected. ${ }^{\star \star} P<0.01 ;{ }^{\star \star \star} P<0.001$. 
IL-1 $\beta$ bioactivity in the mice. Furthermore, to investigate the effect of IL-1 $\beta$ on premalignant neoplasia growth and late-stage tumor progression, two schedules of IL-1Ra injection were used: in the first, IL-1Ra was injected during four cycles of DSS administration, whereas, in the second, it was injected only after the last DSS cycle (Figure 3a).

Using the first schedule, the number of tumor nodules in the colon of mice treated with IL-1Ra was significantly decreased (Figure 3b), mucosal injury and inflammatory infiltrates decreased (Figure 3c), and apoptosis of IECs increased (Figure 3d). Furthermore, nuclear factor $-\kappa \mathrm{B}$ activation in IECs was reduced considerably in IL-1Ra-treated mice (Figure 3e). Using the second schedule (end-stage disease), IL-1Ra treatment resulted in a significant decrease in frequency and size of colon tumors in mice harboring established CAC (Supplementary Figure S5A and B). This effect may be attributed to reduced proliferation as well as increased apoptosis of IECs (Supplementary Figure S5C and D). Overall, these findings indicate an important role of neutrophil-derived IL- $1 \beta$ in CAC promotion and progression.

\section{IL-1 $\beta$ induces IL-6 production by intestinal mononuclear phagocytes (MPs)}

To dissect the mechanisms underlying the protumor function of IL-1 $\beta$, we hypothesized that the effect of IL-1 $\beta$ might be mediated by IL- 6 , a putative CAC-promoting factor, as IL- $1 \beta$ is known to be a strong inducer of IL- 6 production in several cell types. ${ }^{16}$ As expected, IL-6 protein levels in the affected colon of CAC-bearing mice were dramatically increased compared with those in naive mice (Supplementary Figure S6A). Dissection of the cell types responsible for secreting IL-6 in CAC milieus
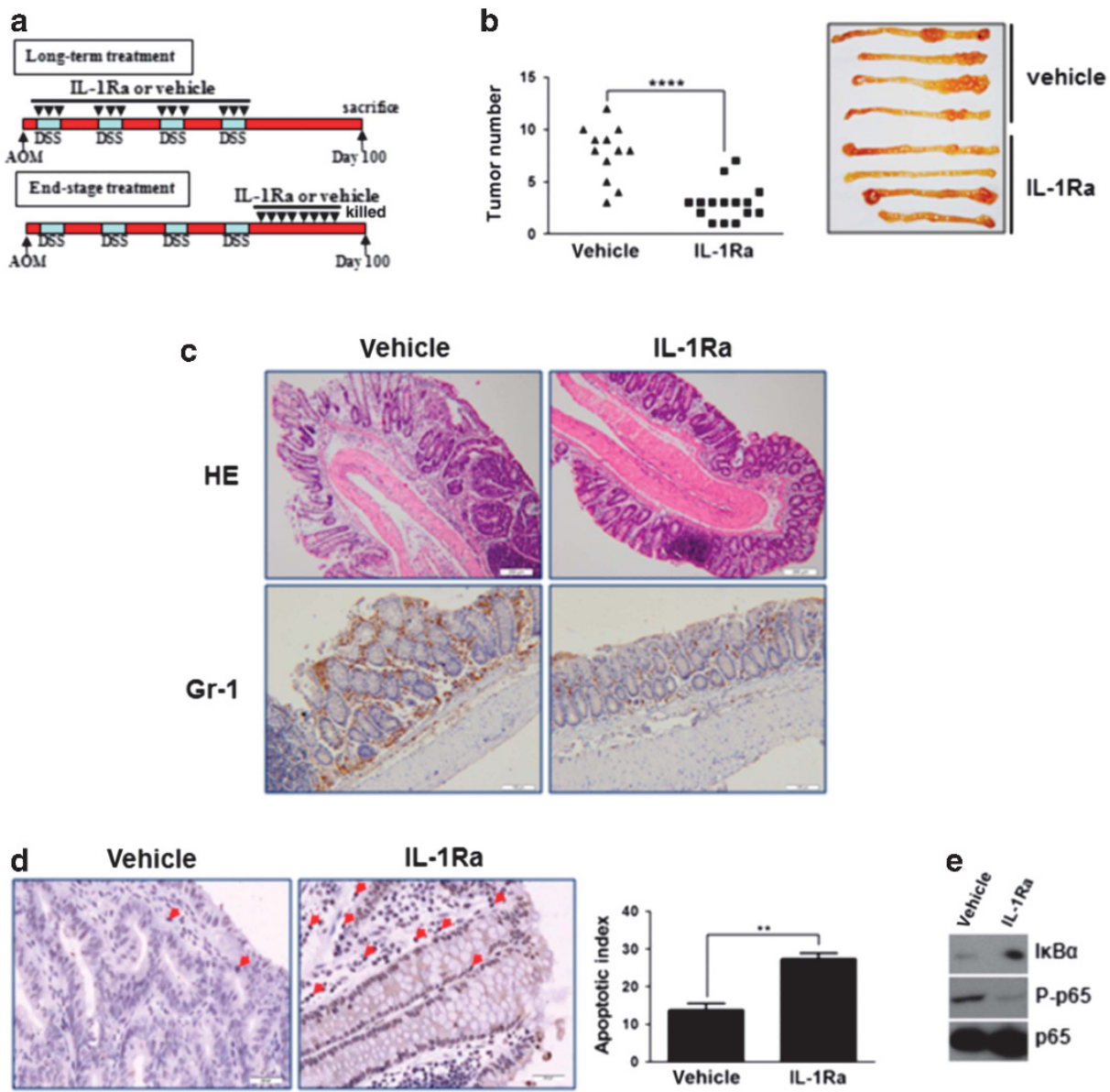

Figure 3 Blockade of interleukin-1 (IL-1) signaling reduces colitis-associated cancer (CAC) tumorigenesis. (a) Diagram showing the protocols for IL-1R antagonist (IL-1Ra) treatment during CAC induction or during late-stage CAC. Mice were injected intraperitoneally with IL-1Ra ( $0.5 \mathrm{mg}$ per mouse) or phosphate-buffered saline (PBS) on days 1,5 , and 8 of each dextran sulfate sodium (DSS) cycle or every day after the last DSS cycle (day 84 ) for 10 days and tumors were examined on day 100. Mice treated during CAC induction were used in b-e. (b) Mice were treated with IL-1Ra or vehicle during CAC induction as shown in $\mathbf{a}$, and then the number of tumors in the colon was counted (left); the right panel shows representative images. (c) The colon tissues were dissected and processed conventionally for hematoxylin and eosin (HE) staining. (d) Colon tissues from mice treated with IL-1Ra or vehicle during CAC induction were isolated and TUNEL (TdT-mediated dNTP nick end labeling) stained (brown) for apoptosis of intestinal epithelial cells (IECs). Six pairs of mice were analyzed. Left: typical result; scale bar $=200 \mu \mathrm{m}$. The arrows denote the apoptotic cells. Right: summarized results. (e) IECs were isolated from the colons of mice treated with IL-1Ra or vehicle during CAC induction and proteins were extracted, and then phosphorylation of the nuclear factor (NF)- $\kappa B$ p65 subunit and $\mathrm{l} \kappa \mathrm{B} \alpha$ levels were measured by western blotting. The data shown are representative of those obtained in three independent experiments. ${ }^{* *} P<0.01 ;{ }^{* * *} P<0.0001$. 
showed that IL-6 mRNA was expressed almost exclusively in DCs and macrophages, i.e., in intestinal MPs (Supplementary Figure S6B). Moreover, IL-6 mRNA expression was considerably higher in DCs than in macrophages, in agreement with a previous study. ${ }^{5}$

To determine whether IL-6 acted downstream of IL-1 signaling, we isolated $\mathrm{Gr}-1^{\mathrm{lo} /-} \mathrm{CD} 11 \mathrm{~b}{ }^{+}$immune cells from the LP of mice with or without IL-1Ra treatment during the DSS cycles for CAC induction (Figure 3a). As shown in Figure 4a, IL-1Ra injection led to a significant reduction in IL-6 mRNA expression by $\mathrm{CD}_{11} \mathrm{~b}^{+}$MPs, showing that IL-1 signaling was needed for IL- 6 production by intestine-resident immune cells during CAC induction in vivo. To evaluate a direct effect of IL$1 \beta$ on IL- 6 production, intestinal CD $11 \mathrm{~b}^{+} \mathrm{MPs}$ were prepared from naive mice and stimulated by IL-1 $\beta$, and the results showed that IL-1 $\beta$ stimulation alone was sufficient to elicit the synthesis of IL-6 mRNA (Figure 4b) and protein (Figure 4c). Furthermore, in coculture experiments, neutrophils isolated from the LP of CAC-bearing mice, but not naive mice, were able to induce IL-6 production in CD11b ${ }^{+}$MPs (Figure 4d) and this effect was prevented by addition of IL-1Ra to the medium. These results suggest that infiltrating neutrophils produce a high level of IL-1 $\beta$ that then induces LP-resident DCs/ macrophages to express IL-6.

\section{Commensal flora translocation is required for IL-1及 production by infiltrated neutrophils}

In our mouse model, commensal microbiota translocation following injury to the epithelial barrier induced by DSS instillation is a key event in triggering intestinal inflammation. ${ }^{17}$ We therefore speculated that invasion by the luminal microflora was necessary for neutrophil infiltration and their increased expression of IL- $1 \beta$. To address this, commensal microorganisms were eradicated by antibiotics and inflammatory infiltrates were examined in the control and antibiotictreated CAC mice and the results showed that antibiotics administration profoundly reduced neutrophil infiltration (Figure 5a). Furthermore, the ability of gut neutrophils to produce IL-1 $\beta$ was significantly impaired (Figure $5 \mathbf{b}$ ). These findings suggest that the signaling triggered by commensal flora-derived ligands is important for IL-1 $\beta$ expression by infiltrating neutrophils. Furthermore, using Toll-like receptor 4 (TLR4)-knockout mice that are protected against CAC as previously reported, ${ }^{18}$ we showed that the absence of TLR4mediated signaling also led to a considerable reduction in IL-1 $\beta$ production by infiltrating neutrophils (Figure $6 \mathbf{c}$ ). We also isolated neutrophils from the spleen of naive mice and stimulated these cells with the TLR4 ligand lipopolysaccharide (LPS), and found that LPS induced IL-1 $\beta$ expression (Figure 6d).
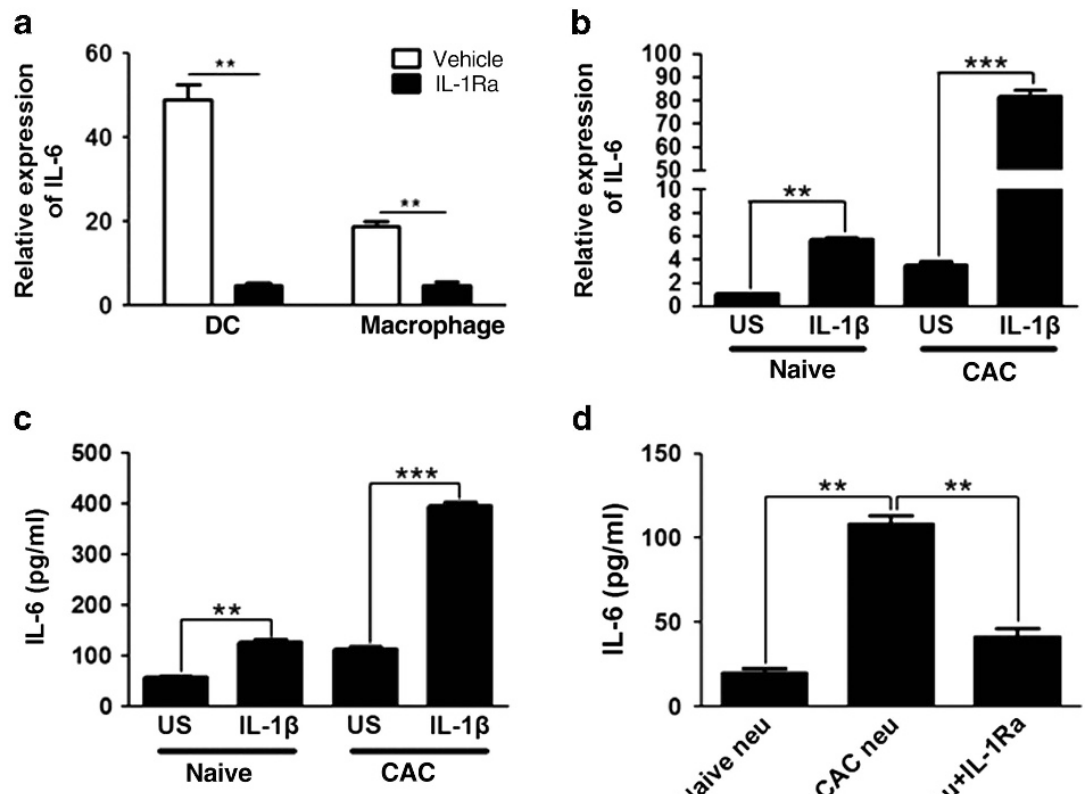

d

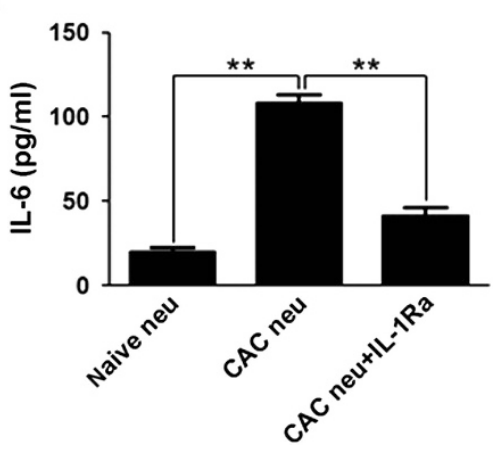

Figure 4 Interleukin-6 (IL-6) secretion by lamina propria (LP) mononuclear phagocytes in colitis-associated cancer (CAC)-bearing mice is regulated by neutrophil-derived IL-1 $\beta$. (a) Dendritic cells (DCs; CD11 $\left.{ }^{+} \mathrm{CD}_{11 b^{+}}\right)$and macrophages $\left(\mathrm{F} 4 / 80^{+} \mathrm{CD} 11 \mathrm{~b}^{+}\right)$in the colon $\mathrm{LP}$ of mice treated with IL-1R antagonist (IL-1Ra) or vehicle during CAC induction were sorted by fluorescence-activated cell sorting (FACS) respectively and IL-6 mRNA levels were determined by quantitative reverse-transcriptase-polymerase chain reaction (RT-PCR). Six pairs of mice were analyzed. (b, $\mathbf{c})$ Mononuclear phagocytes sorted from the colon LP of CAC-bearing or naive littermates were stimulated with IL-1 $\beta\left(1 \mu \mathrm{g} \mathrm{ml}^{-1}\right)$ for $48 \mathrm{~h}$ and then IL-6 expression was measured by (b) quantitative RT-PCR or (c) enzyme-linked immunosorbent assay (ELISA). The data are pooled from three independent experiments. US, unstimulated. (d) Mononuclear phagocytes were sorted from the colon LP of naive mice and cocultured with neutrophils from naive mice or neutrophils from the lesions of CAC-bearing mice for $48 \mathrm{~h}$ with and without IL-1Ra $\left(1 \mu \mathrm{g} \mathrm{ml}^{-1}\right)$ and then IL-6 levels in the supernatants were measured by ELISA. The data are pooled from two independent experiments. ${ }^{* \star} P<0.01 ;{ }^{* \star} P<0.001$. 
a

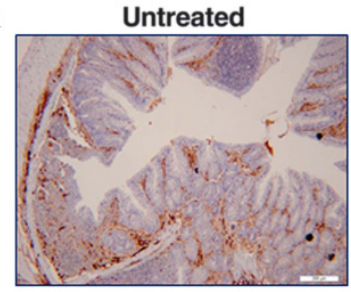

C

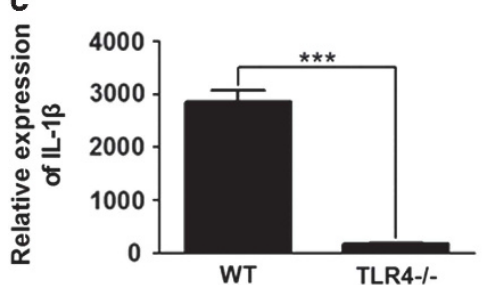

Antibiotics

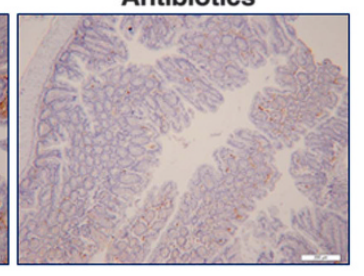

d
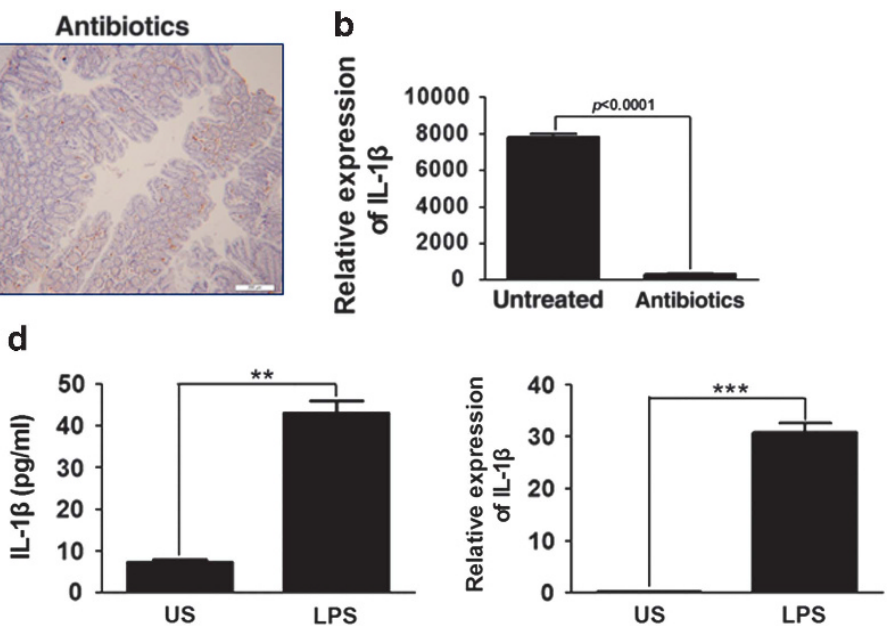

Figure 5 Interleukin-1 $\beta$ (IL-1 $\beta$ ) expression by infiltrating neutrophils in colitis-associated cancer (CAC) lesions is dependent on the commensal flora. (a) Commensal microbiota were left untreated or were eradicated by antibiotic treatment before CAC induction, and then at day 100 of CAC induction, the colons were isolated and stained with anti-Gr-1 antibody for detection of neutrophils. The data shown are representative of those obtained in six sets of mice. (b) Neutrophils infiltrating the colon tissues of mice with or without commensal eradication were isolated and IL-1 $\beta$ mRNA levels were measured by quantitative reverse-transcriptase-polymerase chain reaction (RT-PCR). Five pairs of mice were analyzed. (c) Neutrophils infiltrating the colon of TLR4 - I - or wild-type mice that underwent CAC induction were isolated and IL-1 $\beta$ mRNA levels were measured by quantitative RT-PCR. Five pairs of mice were analyzed. (d) Neutrophils from the spleen of naive mice were isolated by fluorescence-activated cell sorting (FACS) and stimulated with LPS $\left(20 \mathrm{ng} \mathrm{ml}^{-1}\right)$ for $48 \mathrm{~h}$, and then IL-1 $\beta$ protein (left) and mRNA (right) levels were measured by enzyme-linked immunosorbent assay (ELISA) or quantitative RT-PCR. ${ }^{\star \star} P<0.01 ;{ }^{* * \star} P<0.001$.

Overall, these findings indicate a crucial role of commensal flora-derived LPS-mediated signaling in promoting IL-1 $\beta$ expression in infiltrating neutrophils during CAC carcinogenesis.

\section{The IL-1/IL- 6 cascade is involved in development of ulcerative colitis-associated cancer}

To examine whether the results in mice applied to human disease, colon specimens were collected from patients with ulcerative colitis-associated cancer. Immunofluorescence staining for neutrophils (human neutrophil peptides 1-3) and IL-1 $\beta$ showed marked accumulation of IL-1 $\beta$-expressing neutrophils in the inflamed colon tissues of ulcerative colitisassociated cancer patients (Figure 6a). Moreover, fluorescenceactivated cell-sorted infiltrating neutrophils $\left(\mathrm{CD} 6 \mathrm{~b}^{+}\right)$from CAC lesions contained much higher levels of IL-1 $\beta$ mRNA than those from the peripheral blood of healthy volunteers (Figure 6b). In addition, $\mathrm{CD}^{+} 8^{+} \mathrm{MPs}$ in intestinal lesions produced large amounts of IL-6 protein (Figure 6c) and mRNA (Figure 6d). To test the ability of IL-1 $\beta$-expressing neutrophils to induce IL-6 production in DCs/macrophages, we isolated neutrophils from colon tissues of patients with CAC or the peripheral blood of healthy volunteers and cocultured them with intestinal CD $11 b^{+}$MPs from the healthy colon adjacent to the tumor and found that CAC-derived neutrophils induced the control MPs to produce IL-6 protein and that this effect was IL-1 $\beta$ dependent, as it was prevented by addition of the IL-1Ra (Figure 6e). In addition, direct IL-1 $\beta$ stimulation was sufficient to induce IL-6 mRNA expression in LP-derived MPs from healthy colon (Figure 6f), indicating a key function of neutrophil-derived IL- $1 \beta$ in the induction of IL- 6 expression in intestinal MPs. Furthermore, LPS challenge was able to drive control neutrophils to produce IL-1 $\beta$ mRNA (Supplementary Figure S7).

\section{DISCUSSION}

The results of our study provide support for a protumor function of infiltrated neutrophils in carcinogenesis in inflammation-associated colon cancer, although their role in tumor development is still controversial. ${ }^{19-21}$ To examine this issue, Fridlender et al. ${ }^{9}$ recently proposed that, like tumorassociated macrophages, tumor-associated neutrophils can be classified into two subpopulations with an antitumor (N1) or protumorigenic phenotype (N2), differentiation of which is dependent on the tumor microenvironment. Given the tumorpromoting function of neutrophils infiltrating colon tissues of CAC-bearing mice and patients seen in our study, they can be classified as N2 tumor-associated neutrophil.

IL-1 signaling has been shown to play a critical role in gut homeostasis, intestinal inflammation, and colitis-associated tumorigenesis, ${ }^{14}$ but the cellular source of IL-1 $\beta$, an important member of the IL-1 family, has not been determined. Herein, we provide evidence that, in colonic inflammation, IL-1 $\beta$ is predominantly produced by infiltrated neutrophils. Interestingly, a previous study demonstrated that, in the steady state, intestinal macrophages constitutively express IL- $1 \beta$ that is pivotal for $T$ helper type 17 cell differentiation in the intestine. ${ }^{22}$ These findings indicate that the pattern of IL- $1 \beta$ expression in intestinal tissues is regulated by the local microenvironment. It is noteworthy that in our study, a relatively high level of IL-1 $\beta$ mRNA expression was observed in neutrophils in naive mice, but not in $\mathrm{CD} 11 \mathrm{~b}^{+}$phagocytes (Figure 2b). This difference can 
a
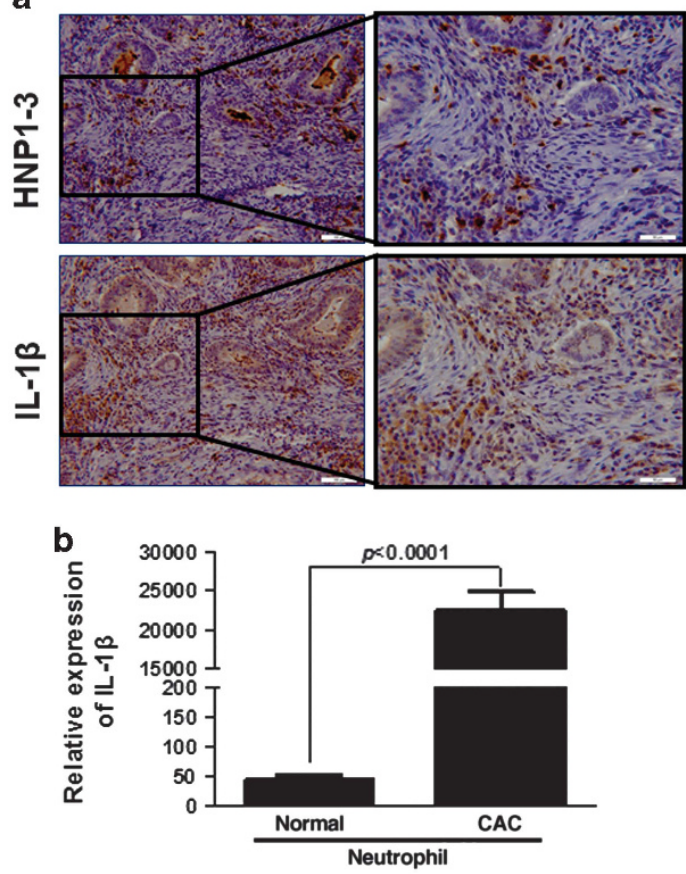

e

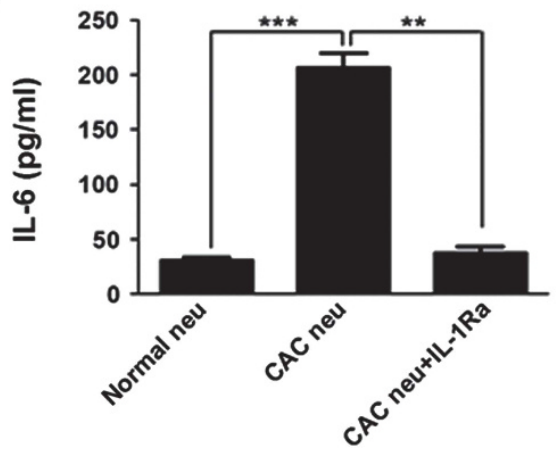

C
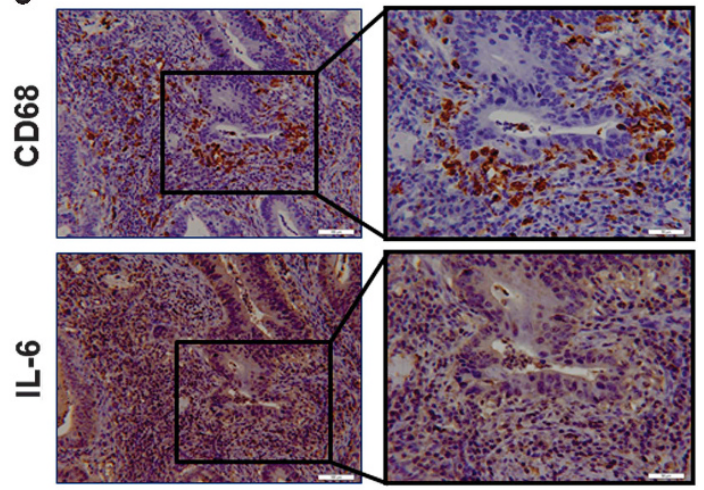

d

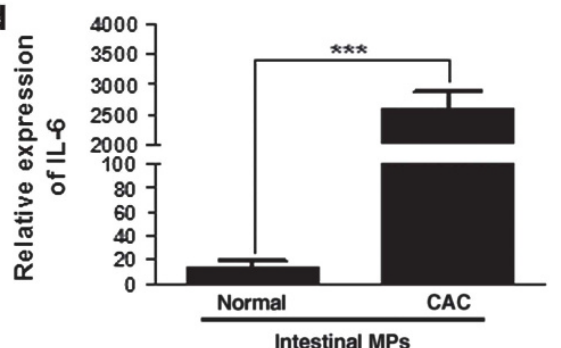

f

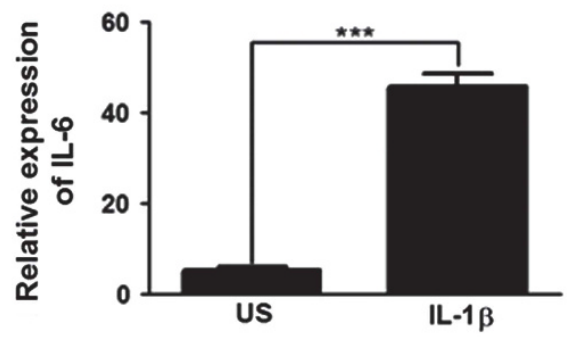

Figure 6 The interleukin (IL)-1/IL-6 axis is involved in the development of ulcerative colitis (UC)-associated colorectal cancer. (a) Immunohistochemical staining for neutrophils (human neutrophil peptide (HNP)1-3-positive) and IL-1 $\beta$ (brown) was performed on formalin-fixed serial sections of surgical colon samples from 18 patients with colitis-associated cancer (CAC). Representative results for one sample are shown. Scale bar $=100 \mu \mathrm{m}$ (left), $50 \mu \mathrm{m}$ (right). (b) Neutrophils $\left(C D 66 b^{+}\right)$infiltrating the lesions of patients with CAC or from the peripheral blood of healthy volunteers were sorted by fluorescence-activated cell sorting (FACS) and IL-1 $\beta$ mRNA levels were measured by quantitative reverse-transcriptase-polymerase chain reaction (RT-PCR). Each group consisted of 5-6 specimens. (c) Surgical colon samples from 15 patients with CAC were collected and intestinal mononuclear phagocytes (MPs; CD68-positive) or IL-6 (brown) detected by immunohistochemistry. Representative results for one sample are shown. Scale bar $=100 \mu \mathrm{m}$ (left), $50 \mu \mathrm{m}$ (right). (d) MPs $\left(\mathrm{CD} 8^{+} \mathrm{CD} 11 \mathrm{~b}^{+}\right)$were isolated from the colon lamina propria (LP) of patients with CAC or from the normal mucosa and IL-6 mRNA levels were measured by quantitative RT-PCR. Each group consisted of 5-6 specimens. (e) Neutrophils isolated from the $\mathrm{LP}$ of the colon of patients with CAC or from the peripheral blood of healthy volunteers were cocultured with control MPs for $24 \mathrm{~h}$ in the presence or absence of IL-1Ra $\left(2 \mu \mathrm{g} \mathrm{ml}^{-1}\right)$ and then IL-6 protein levels were measured by enzyme-linked immunosorbent assay (ELISA). The data are pooled from three independent experiments. (f) MPs were isolated from the LP of the normal mucosa of patients with CAC and incubated for $24 \mathrm{~h}$ with or without (unstimulated (US)) IL-1 $\beta\left(1 \mu \mathrm{g} \mathrm{ml}^{-1}\right.$ ), and then IL-6 expression was measured by quantitative RT-PCR. The data are pooled from three independent experiments. ${ }^{\star *} P<0.01 ;{ }^{\star \star *} P<0.001$.

be explained if, in the homeostatic state, the frequency of neutrophils in the intestine is much lower than that of macrophages and that, upon initiation of colitis, a large number of neutrophils move into the mucosa and release substantial amounts of IL-1 $\beta$ in response to proinflammatory stimuli, similar to a model of LPS-induced shock. ${ }^{23}$ The commensal flora may be an important inducer for IL-1 $\beta$, as, in our study, antibiotic treatment or TLR4 deficiency led to a significant reduction in IL- $1 \beta$ expression by the infiltrated neutrophils. It should be noted that we did not found substantial expression of
IL-1 $\beta$ in spleen neutrophils from CAC-bearing mice, indicating that luminal bacteria translocation is necessary for IL- $1 \beta$ expression by infiltrated neutrophils. In addition, it is noteworthy that although depletion of neutrophil or blockade of IL-1 activity actively repressed CAC formation, these treatments did not completely eradicate the tumors. These data suggest that IL-1 $\beta$-producing neutrophil is a key player in the pathogenesis of CAC, and other components such as IL-21, tumor necrosis factor, and vascular endothelial growth factor also contribute to CAC development, as shown by previous 
reports. ${ }^{24-26}$ Therefore, it is plausible that several pathways including neutrophil/IL-1 $\beta / \mathrm{IL}-6$ axis synergistically orchestrate to promote CAC formation.

It is well established that the inflammasome, a multiprotein complex formed by NOD-like receptor (NLR) family members, converts inactive procaspase- 1 to active caspase- 1 that cleaves the inactive IL-1 in precursor to a secreted and active cytokine. $^{27}$ Intriguingly, mice deficient in inflammasome NLRP3 or caspase-1 have been found to be more susceptible to colitis and CAC. ${ }^{28,29}$ Although it is well established that components of the inflammasome function to process pro-IL$1 \beta$ and IL-18 into mature cytokines, the phenotype of NLRP3 - / - mice with more severe colitis appears not to be attributed to reduced levels of proinflammatory mediators IL-1 $\beta$, given that deficiency in a negative regulator for IL- $1 \mathrm{R}$ signaling renders mice more susceptible to colitis and cancer induction, ${ }^{14,15}$ which is consistent with the conclusions drawn in our study. One possible explanation of enhanced inflammatory reaction in NLRP3 $-/-$ mice can be attributed to reduced IL-18 levels. ${ }^{30,31}$ Furthermore, exogenous IL-18 injection resulted in less weight loss in DSS-instilled caspase-1 - / - mice. ${ }^{30}$ Thus, NLRP3 inflammasome signaling through IL-18 confers protection against DSS-induced colitis. An alternative explanation is that increased susceptibility of NLRP3 - I - mice to colitis may be caused by a role of NLRP3caspase 1-mediated signaling in enhancing programmed cell death. ${ }^{32}$ Specifically, NLRP3-caspase 1-mediated signaling is proapoptotic in infiltrating immune cells during colitis and its deficiency may lead to reduced apoptosis of these cells, and thereby more severe colitis. In addition, it is noteworthy that, in contrast to the conclusions as shown by studies above, Bauer et $a .^{33}$ demonstrated that NLRP3 inflammasome signaling played a pathogenic role during colitis and NLRP3 deficiency conferred protection against mucosal damage during intestinal inflammation. Therefore, further investigations in the roles of inflammasomes in intestinal inflammation are warranted.

With respect to the actions of IL- $1 \beta$, in this study, we show that IL-6 is an important link between IL-1 $\beta$ and CAC formation and that neutrophil-derived IL- $1 \beta$ is a critical inducer of IL- 6 production by intestinal MPs. The CACpromoting effect of IL- 6 has been proposed to be because of its ability to enhance the proliferation of IECs in an inflammatory situation and protect them against apoptosis through the signal transducer and activator of transcription 3-mediated signaling pathway. ${ }^{5,6}$ Therefore, we believe that infiltrating neutrophils produce large amounts of IL- $1 \beta$ in response to flora stimuli and the IL-1 $\beta$ then enhances IL- 6 production in intestine-resident MPs, thereby promoting CAC transformation and growth. The crucial role of IL- $1 \beta$ in intestine homeostasis as a housekeeper that can distinguish harmful from nonpathogenic microorganisms in the gut has been recently highlighted. ${ }^{34}$ Taken together, these findings strongly support a key role of IL- $1 \beta$ in regulating intestinal immunity not only in the homeostatic state, but also in pathogenic states.

In the inflammatory situation, neutrophil recruitment into the intestine is regulated by a series of cytokines and chemokines. ${ }^{35}$ We have previously demonstrated the expression of several factors in the mucosa and found that, during DSS-induced colitis, there is a significant increase in the expression of several chemokines, ${ }^{36}$ including CXCL2, that have well established roles in recruiting peripheral neutrophils into tumor sites. ${ }^{37}$ Intriguingly, IL-1 $\beta$ has an indirect role in recruiting granulocytes. ${ }^{38}$ In fact, blockade of IL-1 $\beta$ activity in our study led to a significant reduction in neutrophils in the colon mucosa, indicating a positive feedback of IL- $1 \beta$ on the recruitment of more neutrophils into colonic tissues, thereby exacerbating the inflammatory response. Overall, our study provides a mechanistic explanation of how neutrophils are involved in the development of inflammation-related colorectal cancer. Neutrophil-targeted therapy or blockade of IL-1 $\beta$ bioactivity using specific antagonists may be beneficial for the treatment of CAC in the clinic.

\section{METHODS}

Mice. Balb/c, TLR4 $-/-$, and nude mice were purchased from Jackson Laboratory (Bar Harbor, ME) and housed in specific pathogenfree conditions on an alternating light/dark cycle. TLR $4-/-\mathrm{Balb} / \mathrm{c}$ mice were obtained by backcross for 12 generations onto the Balb/c background; in this study, all references to 'TLR4 - / -' or 'TLR4 knockout' mice refer to these backcrossed mice. All animal experiments were performed in accordance with international guidelines for the care and use of laboratory animals and approved by the Animal Ethics Committee of the Institute of Basic Medical Sciences.

Patients. Freshly isolated neoplastic tissue samples and adjacent normal mucosa were obtained from patients undergoing surgical resection with the diagnosis of CAC $(n=18)$. No chemotherapy or radiotherapy was performed before collection of the tissue samples. The samples were sectioned for immunohistochemistry and hematoxylin and eosin staining and for the isolation of LP immune cells. Prior informed consent was obtained and the study protocol was approved by the Ethics Committee of the General Hospital of the PLA (Beijing, China).

CAC model. CAC was induced as described previously, ${ }^{39}$ with slight modifications. Male Balb/c or TLR4 - / - mice aged 6-8 weeks were injected once intraperitoneally with azoxymethane $\left(10 \mathrm{mg} \mathrm{kg}^{-1}\right.$; Sigma-Aldrich, St Louis, MO) and maintained on regular diet and water for 5 days. They then underwent the first DSS treatment cycle in which they received drinking water containing 2\% DSS (molecular weight 36,000-50,000; MP Biochemicals, Solon, OH) for 1 week, starting on day 0 , then plain water for 2 weeks ( 1 cycle), and this was followed by three more DSS treatment cycles ( 84 days total). They were then maintained on a regular diet with normal drinking water until day 100 , when they were killed and macroscopic tumors counted. The clinical course of the disease was followed daily by measurement of body weight and monitoring for signs of rectal bleeding or diarrhea.

Histopathology and immunohistochemistry. The protocol on histopathology and immunohistochemistry is described in Supplementary Materials and Methods.

Immunoblotting. Standard procedures for immunoblotting are described in Supplementary Materials and Methods.

Mouse whole colon cultures. The experiments were done as described in Supplementary Materials and Methods.

Detection and quantification of apoptotic cells. Experiments were carried out as described in Supplementary Materials and Methods. 
In vivo antibody or antagonist treatment. Experiments were performed as described in Supplementary Materials and Methods.

Isolation of mouse and human colonic epithelial cells. Colonic epithelial cells were fractionated as described previously ${ }^{40}$ with light modification. The details are described in Supplementary Materials and Methods.

Isolation of mouse spleen cells, mouse and human LP immune cells, human blood neutrophils, and in vitrotreatment. The protocol is described in Supplementary Materials and Methods.

Flow cytometry. The experiments were done as described in Supplementary Materials and Methods.

Commensal flora depletion. The protocol of depletion of Commensal microbiota was detailed in Supplementary Materials and Methods. The efficacy of commensal depletion by the antibiotics was confirmed by staining with SYBR green solution (TOYOBO, Osaka, Japan) as described previously. ${ }^{14}$

Tumor transplantation. Experiments were performed as described in Supplementary Materials and Methods.

Cytokine assays. IL- 6 and IL- $1 \beta$ levels in culture supernatants were measured using enzyme-linked immunosorbent assay kits (R\&D Systems, Minneapolis, MN) according to the manufacturer's instructions.

Quantitative reverse-transcriptase-polymerase chain reaction. Experiments were carried out as described in Supplementary Materials and Methods.

Statistical analysis. Statistical analysis was performed as described in Supplementary Materials and Methods.

SUPPLEMENTARY MATERIAL is linked to the online version of the paper at http://www.nature.com/mi

\section{ACKNOWLEDGMENTS}

We thank Dr Zhihai Qin for providing the CT26 cell line and Dr Haiyan Hong for providing the IL-1Ra. This work was supported by grants from the National Key Basic Research Program of China (2013CB530506), the National Natural Science Foundation of China $(81272320,81072475)$, and the Beijing Natural Science Foundation (7132151).

\section{DISCLOSURE}

The authors declared no conflict of interest.

c) 2014 Society for Mucosal Immunology

\section{REFERENCES}

1. Mantovani, A., Allavena, P., Sica, A. \& Balkwill, F. Cancer-related inflammation. Nature 454, 436-444 (2008).

2. Rutter, M. et al. Severity of inflammation is a risk factor for colorectal neoplasia in ulcerative colitis. Gastroenterology 126, 451-459 (2004).

3. Gupta, R.B. et al. Histologic inflammation is a risk factor for progression to colorectal neoplasia in ulcerative colitis: a cohort study. Gastroenterology 133, 1099-1105 (2007).

4. Erreni, M., Mantovani, A. \& Allavena, P. Tumor-associated macrophages (TAM) and inflammation in colorectal cancer. Cancer Microenviron. 4, 141-154 (2011).

5. Grivennikov, S. et al. IL-6 and stat3 are required for survival of intestinal epithelial cells and development of colitis-associated cancer. Cancer Cell 15, 103-113 (2009).

6. Matsumoto, S. et al. Essential roles of IL-6 trans-signaling in colonic epithelial cells, induced by the IL-6/soluble-IL-6 receptor derived from lamina propria macrophages, on the development of colitis-associated premalignant cancer in a murine model. J. Immunol. 184, 1543-1551 (2010).
7. Fridlender, Z.G. \& Albelda, S.M. Tumor-associated neutrophil: friend or foe? Carcinogenesis 33, 949-955 (2012).

8. Gregory, A.D. \& Houghton, A.M. Tumor-associated neutrophil: new targets for cancer therapy. Cancer Res. 71, 2411-2416 (2011).

9. Fridlender, Z.G. et al. Polarization of tumor-associated neutrophil phenotype by TGF- $\beta$ : 'N1' versus 'N2' TAN. Cancer Cell 16, 183-194 (2009).

10. Vermeer, I.T. et al. Neutrophil-mediated formation of carcinogenic $\mathrm{N}$-nitroso compounds in an in vitro model for intestinal inflammation. Toxicol. Lett. 154, 175-182 (2004).

11. Shang, K. et al. Crucial involvement of tumor-associated neutrophils in the regulation of chronic colitis-associated carcinogenesis in mice. PLoS One 7, e51848 (2012).

12. Daley, J.M., Thomay, A.A., Connolly, M.D., Reichner, J.S. \& Albina, J.E. Use of Ly6G-specific monoclonal antibody to deplete neutrophil in mice. J. Leukoc. Biol. 83, 64-70 (2008).

13. Rakoff-Nahoum, S., Paglino, J., Eslami-Varzaneh, F., Edberg, S. \& Medzhitov, R. Recognition of commensal microflora by toll-like receptors is required for intestinal homeostasis. Cell 118, 229-241 (2004).

14. Xiao, H. et al. The Toll-Interleukin-1 receptor member SIGIRR regulates colonic epithelial homeostasis, inflammation, and tumorigenesis. Immunity 26, 461-475 (2007).

15. Garlanda, C. et al. Increased susceptibility to colitis-associated cancer of mice lacking TIR8, an inhibitory member of the interleukin-1 receptor family. Cancer Res. 67, 6017-6021 (2007).

16. Dinarello, C.A. Biological basis for interleukin-1 in disease. Blood 87, 2095-2147 (1996).

17. Sussman, D.A., Santaolalla, R., Strobel, S., Dheer, R. \& Abreu, M.T. Cancer in inflammatory bowel disease: lessons from animal models. Curr. Opin. Gastroenterol. 28, 327-333 (2012).

18. Fukata, M. et al. Toll-like receptor-4 promotes the development of colitisassociated colorectal tumors. Gastroenterology 133, 1869-1881 (2007).

19. Kousis, P.C., Henderson, B.W., Maier, P.G. \& Gollnick, S.O. Photodynamic therapy enhancement of antitumor immunity is regulated by neutrophils. Cancer Res. 67, 10501-10510 (2007).

20. Suttmann, $H$. et al. Neutrophil granulocytes are required for effective Bacillus Calmette-Guerin immunotherapy of bladder cancer and orchestrate local immune responses. Cancer Res. 66, 8250-8257 (2006).

21. Mittendorf, E.A. et al. Breast cancer cell uptake of the inflammatory mediator neutrophil elastase triggers an anticancer adaptive immune response. Cancer Res. 72, 3153-3162 (2012).

22. Shaw, M.H., Kamada, N., Kim, Y.G. \& Núñez, G. Microbiota-induced IL-1ß, but not IL-6, is critical for the development of steady-state $T_{H} 17$ in the intestine. J. Exp. Med. 209, 251-258 (2012).

23. Greten, F.R. et al. NF-KB is a negative regulator of $\mathrm{IL}-1 \beta$ secretion as revealed by genetic and pharmacological inhibition of IKK $\beta$. Cell 130, 918-931 (2007).

24. Stolfi, C. et al. Involvement of interleukin-21 in the regulation of colitisassociated colon cancer. J. Exp. Med. 208, 2279-2290 (2011).

25. Popivanova, B.K. et al. Blocking TNF- $\alpha$ in mice reduces colorectal carcinogenesis associated with chronic colitis. J. Clin. Invest. 118, 560-570 (2008).

26. Waldner, M.J. et al. VEGF signaling links inflammation and tumorigenesis in colitis-associated cancer. J. Exp. Med. 207, 2855-2868 (2010).

27. Lopez-Castejon, G. \& Brough, D. Understanding the mechanism of IL-1 $\beta$ secretion. Cytokine Growth Factor Rev. 22, 189-195 (2011).

28. Allen, I.C. et al. The NLRP3 inflammasome functions as a negative regulator of tumorigenesis during colitis-associated cancer. J. Exp. Med. 207, 1045-1056 (2010).

29. Hirota, S.A. et al. The NLRP3 inflammasome plays key role in the regulation of intestinal homeostasis. Inflamm. Bowel Dis. 17, 1359-1372 (2011).

30. Zaki, M.H. et al. The NLRP3 inflammasome protects against loss of epithelial integrity and mortality during experimental colitis. Immunity $\mathbf{3 2}$, 379-391 (2010).

31. Dupaul-Chicoine, J. et al. Control of intestinal homeostasis, colitis, and colitis-associated colorectal cancer by inflammatory caspases. Immunity 32, 367-378 (2010).

32. Miura, M., Zhu, H., Rotello, R., Hartwieg, E.A. \& Yuan, J. Induction of apoptosis in fibroblasts by IL-1 beta-converting enzyme, a mammalian homolog of the C. elegans cell death gene ced-3. Cell 75, 653-660 (1993) 
33. Bauer, C. et al. Colitis induced in mice with dextran sulfate sodium (DSS) is mediated by the NLRP3 inflammasome. Gut 59, 1192-1199 (2010).

34. Franchi, L. et al. NLRC4-driven production of IL-1 $\beta$ discriminates between pathogenic and commensal bacteria and promotes host intestinal defense. Nat. Immunol. 13, 449-456 (2012).

35. Kobayashi, Y. The role of chemokines in neutrophil biology. Front. Biosci. 13, 2400-2407 (2008).

36. Wang, K. et al. Opposite role of tumor necrosis factor receptors in dextran sulfate sodium-induced colitis in mice. PLoS One 7, e52924 (2012).
37. Fridlender, Z.G. etal. Transcriptomic analysis comparing tumor-associated neutrophils with granulocytic myeloid-derived suppressor cells and normal neutrophils. PLoS One 7, e31524 (2012).

38. Ross, R. Atherosclerosis is an inflammatory disease. Am. Heart J. $\mathbf{1 3 8}$ S419-S420 (1999).

39. Wirtz, S., Neufert, C., Weigmann, B. \& Neurath, M.F. Chemically induced mouse models of intestinal inflammation. Nat. Protoc. 2, 541-546 (2007).

40. Guo, J., Longshore, S., Nair, R. \& Warner, B.M. Retinoblastoma protein (pRb), but not p107 or p130, is required for maintenance of enterocyte quiescence and differentiation in small intestine. J. Biol. Chem. 284, 134-140 (2009). 